\title{
High-frequency slowly drifting structures and X-ray sources observed by RHESSI
}

\author{
M. Karlický1 ${ }^{\text {, F. Fárník }}{ }^{1}$, and S. Krucker ${ }^{2}$ \\ 1 Astronomical Institute of the Academy of Sciences of the Czech Republic, 25165 Ondřejov, Czech Republic \\ 2 Space Sciences Lab. University of California, Berkeley, USA
}

Received 7 December 2003 / Accepted 10 February 2004

\begin{abstract}
Three solar flares (April 4, 2002, May 17, 2002, and August 30, 2002) with the 0.4-2.0 GHz slowly drifting structures were selected and analyzed together with RHESSI X-ray observations. Two events (April 4, 2002 and May 17, 2002) were observed above and one event (August 30, 2002) close to the solar limb. While in April 4, 2002 and August 30, 2002 the radio drifting structures with relatively high frequency drifts $\left(-32--25 \mathrm{MHz} \mathrm{s}^{-1}\right)$ were recorded at times of the start of a motion of the X-ray flare source, in May 17, 2002 event a splitting of the X-ray source into two sources was observed before observation of the $0.8-1.8 \mathrm{GHz}$ radio structure drifting with very slow frequency drift $\left(-0.4 \mathrm{MHz} \mathrm{s}{ }^{-1}\right)$. The X-ray source of the May 17, 2002 was much softer ( $<40 \mathrm{keV})$ than those in April 4, 2002 and August 30, $2002(>100 \mathrm{keV})$. Velocities of the $X$-ray sources in the image plane were estimated as $12 \mathrm{~km} \mathrm{~s}^{-1}$ for April 4, 2002 and $10 \mathrm{~km} \mathrm{~s}^{-1}$ for August 30, 2002. Analyzing GOES data and X-ray RHESSI spectra of the May 17, 2002 flare the plasma thermal and non-thermal electron densities in the X-ray sources were determined. For two cases (April 4, 2002 and May 17, 2002) it was found that the plasma density in the coronal X-ray source is higher than maximum one derived from the radio drifting structure. The cross-correlation of the radio drifting structure and hard X-ray flux for the August 30, 2002 event reveals that the hard X-ray emission is delayed 0.5-0.7 s after the radio and it is partly correlated with an enhanced background of the drifting structure. All these results are discussed and interpreted considering the flare model with the plasmoid ejection.
\end{abstract}

Key words. Sun: flares - Sun: radio radiation - Sun: X-rays, gamma rays

\section{Introduction}

Slowly drifting radio structures, observed in the $1.5-0.6 \mathrm{GHz}$ frequency range at the beginning of the eruptive solar flares, were found to be a radio signature of a plasmoid ejection (Karlický \& Odstrčil 1994; Karlický 1998; Hori 1999; Khan et al. 2002; Karlický et al. 2002).

Based on the MHD numerical simulations, Kliem et al. (2000) suggested that every individual burst in the slowly drifting structure is generated by superthermal electrons, accelerated in a peak of the electric field in a quasi-periodic and bursting regime of the magnetic field reconnection. Furthermore, the global slow negative frequency drift of the structure was explained by a plasmoid propagation upwards in the solar corona towards lower plasma densities.

Hudson et al. (2001) identified a rapidly moving hard X-ray source, observed by the Yohkoh/HXT, associated with the moving microwave source and the plasmoid ejection seen in the Yohkoh/SXT images. The association with the high-frequency slowly drifting burst was also reported.

Send offprint requests to: M. Karlický, e-mail: karlicky@asu.cas.cz
Similarly, Kundu et al. (2001) identified two moving YOHKOH soft X-ray ejecta associated with moving decimetric/metric radio sources observed by Nancay radioheliograph.

In our last paper in this field we have presented a unique series of high-frequency slowly drifting structures observed during one flare (Karlický 2004). It was proposed that this series of radio bursts maps the magnetic field reconnection in the extended current sheet, i.e. it maps a formation of several plasmoids and their interactions.

Observations made by new space experiment RHESSI (Lin et al. 2002) give us new possibilities in the study of these processes. Namely, positions, sizes and spectral characteristics of X-ray sources (plasmoids) can be determined with higher precision than in previous experiments (e.g. Yohkoh). Therefore in this paper, using new RHESSI observations at times of relatively rare slowly drifting structures, motions of X-ray sources, electron plasma and electron non-thermal densities in the X-ray sources and their relationships to radio drifting structures are studied in details.

\section{The April 4, 2002 event}

During April 4, 2002, GOES satellite registered at 15:24 UT (start) - 15:32 UT (maximum) - 15:38 UT (end) a soft X-ray 
Table 1. Basic characteristics of the studied events and of their slowly drifting structures (DS) in the 0.4-2.0 GHz frequency range and hard $\mathrm{X}$-ray source motions.

\begin{tabular}{|c|c|c|c|}
\hline Event & Apr. 4, 2002 & May 17, 2002 & Aug. 30, 2002 \\
\hline GOES start (UT) & $15: 24$ & $7: 27$ & $12: 47$ \\
\hline GOES maximum (UT) & $15: 32$ & $7: 54$ & $13: 29$ \\
\hline GOES end (UT) & $15: 38$ & $8: 40$ & $13: 35$ \\
\hline GOES class & M 6.1 & M 1.5 & X 1.5 \\
\hline $\mathrm{H} \alpha$ start (UT) & - & - & $12: 26$ \\
\hline $\mathrm{H} \alpha$ maximum (UT) & - & - & $13: 28$ \\
\hline $\mathrm{H} \alpha$ end (UT) & - & - & $13: 49$ \\
\hline $\mathrm{H} \alpha$ class & - & - & $\mathrm{SN}$ \\
\hline NOAA active region & - & - & 0095 \\
\hline Location & source above limb & source above limb & N15 E74 \\
\hline \multicolumn{4}{|l|}{ Drifting structure } \\
\hline \multirow[t]{2}{*}{ Start (UT) } & $15: 28: 48$ & $7: 37$ & $13: 27: 38$ \\
\hline & & $7: 32$ (start of $3 \mathrm{GHz}$ burst) & \\
\hline End (UT) & $15: 29: 20$ & $7: 57$ & $13: 27: 50$ \\
\hline Frequency range $(\mathrm{GHz})$ & $0.45-1.4$ & $0.8-1.8$ & $0.8-2.0$ \\
\hline $\begin{array}{l}\text { Global Freq. drift } \\
\left(\mathrm{MHz} \mathrm{s}^{-1}\right)\end{array}$ & -32 & -0.4 & -25 \\
\hline $\begin{array}{l}\text { Drift of individual pulses } \\
\qquad\left(\mathrm{MHz} \mathrm{s}^{-1}\right)\end{array}$ & infinite & $240-360$ & infinite \\
\hline $\begin{array}{c}\text { Instantaneous } \\
\text { bandwidth }(\mathrm{MHz})\end{array}$ & 200 & 800 & 800 \\
\hline \multicolumn{4}{|l|}{$\mathrm{X}$-ray source motion } \\
\hline Start (UT) & $15: 28$ & $7: 32$ & $13: 28$ \\
\hline End (UT) & $>15: 38$ & $7: 36$ & $>13: 33$ \\
\hline Velocity direction & east & source splitting & north-east \\
\hline$\left(\mathrm{km} \mathrm{s}^{-1}\right)$ & 12 & up to 100 (during the splitting) & 10 \\
\hline
\end{tabular}

event classified as M6.1 flare. No optical flare has been reported at the time of this event (see Table 1).

On radio waves the flare starts with a group of type III bursts at 15:27:42-15:27:52 UT in the $0.8-1.3 \mathrm{GHz}$ frequency range followed in the same frequency range by type III bursts at 15:28:18-15:28:28 UT (Ondřejov radiospectrograph observations). Then the high-frequency slowly drifting structure (DS) was observed at 15:28:48-15:29:20 UT in the frequency range 1.4-0.45 GHz (Fig. 1) (see also Kane et al. 2003). Its frequency drift was estimated as $-32 \mathrm{MHz} \mathrm{s}^{-1}$ (Table 1). Then at 15:29:20-15:30:30 UT the DS stopped to drift. At this time it was accompanied by a broadband continuum in the frequency range $0.6-1.5 \mathrm{GHz}$, by the GOES soft X-ray and RHESSI hard $\mathrm{X}$-ray (40-100 keV) emission increase and by a hardening of the spectral X-ray power-law index (Fig. 1).

As shown in Fig. 2, where the composite EIT/SOHORHESSI image is presented, RHESSI imager observed at 15:29:11-15:30:17 UT a double hard X-ray source just at the solar limb, at positions of the flare loop footpoins (see the EIT/SOHO image observed at 15:36:10 UT with the substraction of that at 14:36:12 UT). On the other hand, in the thermal range of energies $(6.2-7.2 \mathrm{keV})$ only one compact X-ray source at the position of the northern hard X-ray source can be seen. But at the time of observation of the first group of type III bursts this source started to move in the eastward direction with the velocity of about $12 \mathrm{~km} \mathrm{~s}^{-1}$ (see Fig. 3, where also the RHESSI $6.2-7.2 \mathrm{keV}$ light curve and type III bursts and the drifting structure - DS are superposed).

RHESSI hard X-ray spectrum at times of the slowly drifting structure was analyzed (Fig. 4). The best fit was obtained using two temperature plus broken power-law distribution model. The results of the fitting are summarized in Table 2 . The powerlaw index of the non-thermal electron distribution was 3.69 at 15:29:45-15:29:52 UT. The emission measure, temperature of the X-ray thermal source, and corresponding plasma densitities were derived from the GOES as well as from RHESSI measurements using the thermal source size $6 \pm 1$ arcsecs. For comparison the maximum electron plasma density derived from the maximum frequency of the slowly drifting structure, assuming the plasma radiation on the fundamental frequency is added $\left(n_{\mathrm{e}}^{\text {Rad,max }}=2.42 \times 10^{10} \mathrm{~cm}^{-3}\right)$.

\section{The May 17, 2002 event}

During May 17, 2002, GOES satellite observed at 7:27 UT (start) - 7:54 UT (maximum) - 8:40 UT (end) the soft X-ray 


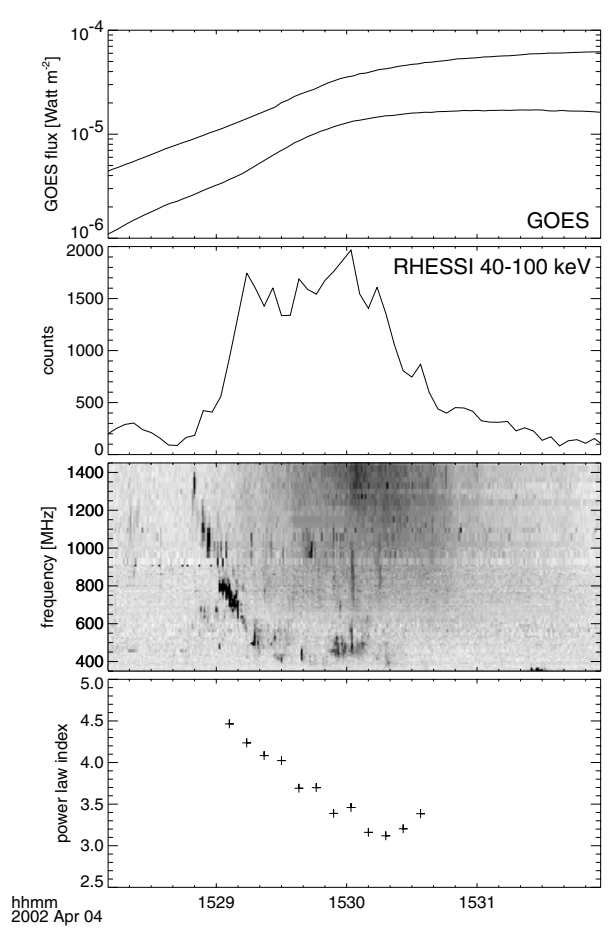

Fig. 1. Time evolution of the April 4, 2002 flare: GOES soft X-ray flux, RHESSI $40-100 \mathrm{keV}$ counts, the $0.4-1.5 \mathrm{GHz}$ radio spectrum showing the slowly drifting structure observed by the Zürich radiospectrograph, and the power-law index derived from RHESSI observations.

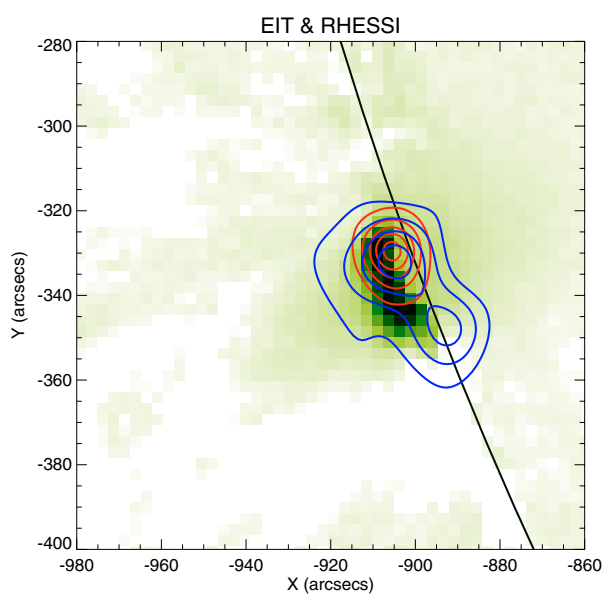

Fig. 2. The combined RHESSI-EIT/SOHO image: the full line contours (levels $=30,50,70,90 \%$ ) show the 50-100 keV RHESSI source at 15:29:11-15:30:10 UT, grey full line contours (levels $=10,30,50$, $70,90 \%$ ) the $6.2-7.2 \mathrm{keV}$ RHESSI source at 15:29:11-15:30:17 UT, both superposed on the EIT/SOHO image observed at 15:36:10 UT, but after the substraction of that from 14:36:12 UT.

event classified as M 1.5 flare. No optical flare has been reported at the time of this event.

On radio waves the event started at $3 \mathrm{GHz}$ at 7:32 UT (Fig. 5, compare also with the HXRS (Fárník et al. 2001) and RHESSI) followed by the high-frequency slowly drifting structure recorded at 7:37-7:57 UT in the frequency range $1.8-0.8 \mathrm{GHz}$ (Fig. 5). Its frequency drift was estimated as $-0.4 \mathrm{MHz} \mathrm{s}^{-1}$ (Table 1). Some individual pulses

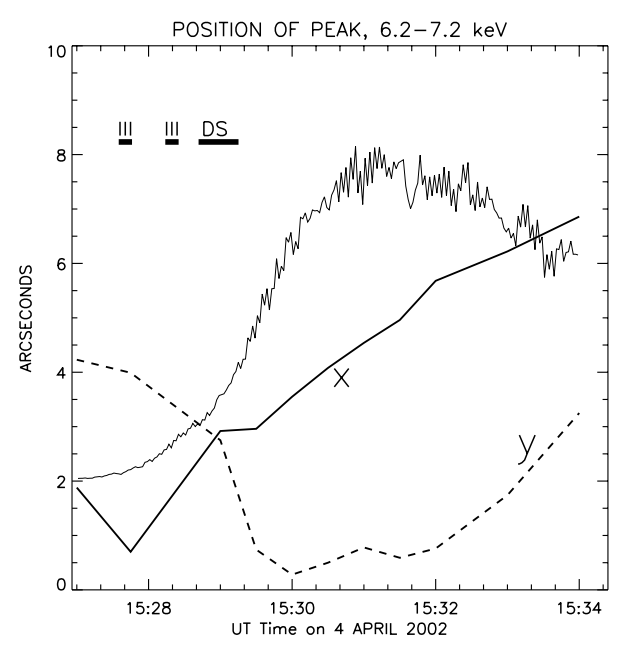

Fig. 3. The east-west ( $x$-full line) and north-south ( $y$-dashed line) positional changes of the peak of the $6.2-7.2 \mathrm{keV}$ source. The zero values correspond to position $x=-902 \operatorname{arcsec}, y=-329 \operatorname{arcsec}-$ see Fig. 2. The positive values mean those in the east and south directions from the reference (zero) point. The RHESSI 6.2-7.2 keV light curve (thin line) is superimposed for comparison (in arbitrary units). Two groups of type III radio bursts and the drifting structure (DS) periods are shown.

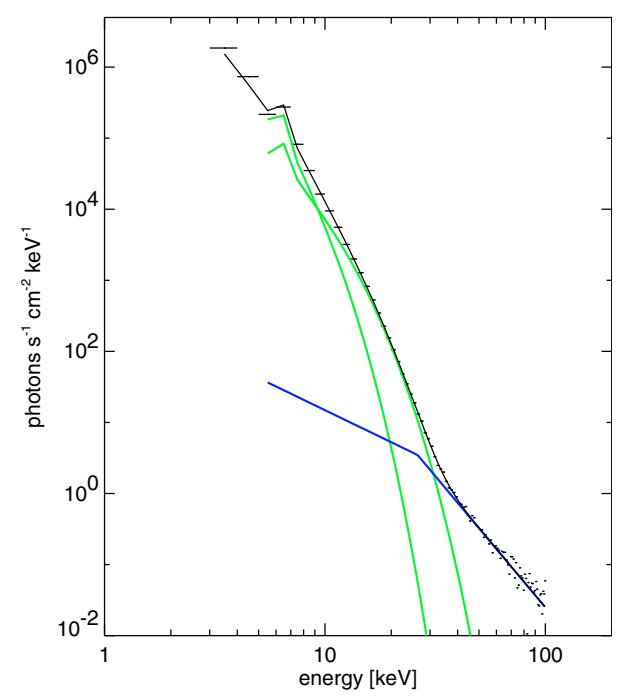

Fig. 4. The RHESSI X-ray spectrum of the hard X-ray source observed during the April 4, 2002 flare at 15:29:45-15:29:53 UT. This spectrum was fitted using the two temperature plus power-law model of the hard X-ray emission. The resulting spectrum (fitting observed data $=1 \mathrm{keV}-$ long horizontal lines) is a sum of the three components: two thermal components (thin lines) and one non-thermal component (thick line).

of this structure had positive frequency drifts in the range $240-360 \mathrm{MHz} \mathrm{s}^{-1}$.

This radio burst was followed later on by the type II radio burst in the frequency range $300-40 \mathrm{MHz}$ at 8:02-8:25 UT (Potsdam-Tremsdorf observation).

RHESSI imager observed the X-ray source $(6-15 \mathrm{keV})$ above the east-north solar limb (Fig. 6) which exhibited an interesting evolution at 7:30-7:36 UT, i.e. at times between the beginning of the $3 \mathrm{GHz}$ radio burst and X-rays (see Fig. 5), 


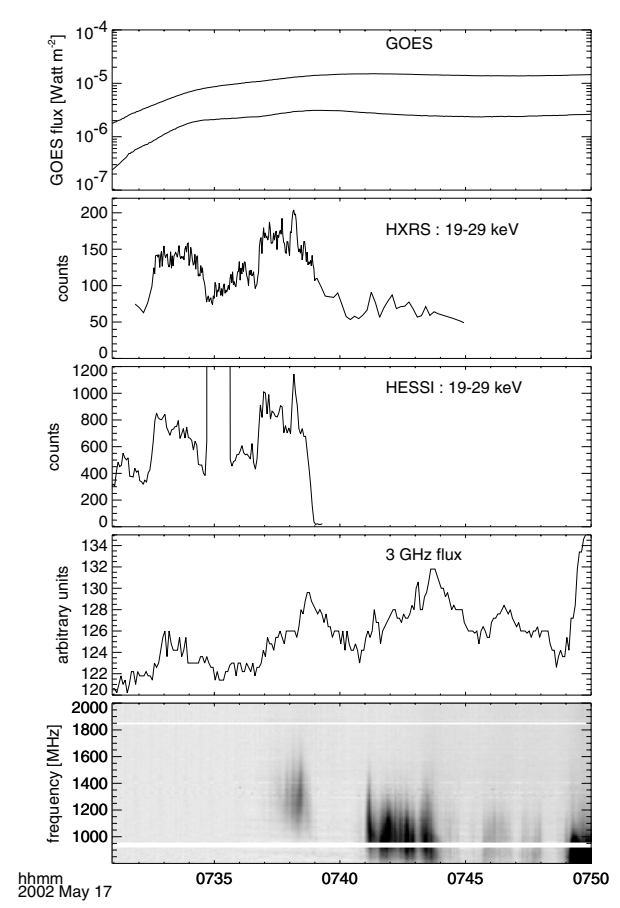

Fig. 5. Time evolution of the May 17, 2002 flare: GOES soft X-ray flux, HXRS 19-29 keV counts, RHESSI 19-29 keV counts, and the $0.8-2.0 \mathrm{GHz}$ radio spectrum showing the slowly drifting structure observed by the Ondřejov radiospectrograph.

Table 2. Parameters derived from GOES data and RHESSI X-ray spectra at the times of the April 4, 2002 drifting structure observations, $n_{\mathrm{e}}^{\mathrm{G}}$ is the electron plasma density derived from the GOES emission measure and the RHESSI source volume, $n_{\mathrm{e}}^{\mathrm{R}} 1$ is the electron plasma density derived from the RHESSI emission measure for the temperature 1 and the RHESSI source volume, $n_{\mathrm{e}}^{\mathrm{R}} 2$ is the electron plasma density derived from the RHESSI emission measure for the temperature 2 and the RHESSI source volume, $n_{\mathrm{e}}^{\text {Rad,max }}$ is the electron plasma density derived from the radio spectrum.

\begin{tabular}{lc}
\hline \hline Time $(\mathrm{UT})$ & $15: 29: 45-: 52$ \\
GOES Emission measure $\left(10^{49} \mathrm{~cm}^{-3}\right)$ & 1.0 \\
GOES Temperature $\left(10^{6} \mathrm{~K}\right)$ & 22 \\
\hline RHESSI fit interval $(\mathrm{keV})$ & $4-100$ \\
RHESSI Emission measure $1\left(10^{49} \mathrm{~cm}^{-3}\right)$ & 3.15 \\
RHESSI Temperature $1\left(10^{6} \mathrm{~K}\right)$ & 18.3 \\
RHESSI Emission measure $2\left(10^{49} \mathrm{~cm}^{-3}\right)$ & 0.28 \\
RHESSI Temperature $2\left(10^{6} \mathrm{~K}\right)$ & 36.0 \\
Thermal source size $(\operatorname{arcsecs})$ & $6 \pm 1$ \\
Thermal source volume $\left(10^{25} \mathrm{~cm}^{3}\right)$ & 8.23 \\
$n_{\mathrm{e}}^{\mathrm{G}}(\mathrm{GOES})\left(10^{11} \mathrm{~cm}^{-3}\right)$ & 3.48 \\
$n_{\mathrm{e}}^{\mathrm{R}}$ (RHESSI $\left(10^{11} \mathrm{~cm}^{-3}\right)$ & 6.18 \\
$n_{\mathrm{e}}^{\mathrm{R}} 2(\mathrm{RHESSI})\left(10^{11} \mathrm{~cm}^{-3}\right)$ & 1.84 \\
$n_{\mathrm{e}}^{\text {Rad,max }}\left(10^{10} \mathrm{~cm}^{-3}\right)$ & 2.42 \\
\hline
\end{tabular}

and start of the slowly drifting structure. (Remark: before the observation of the drifting structure no radio emission was observed in the frequency range below $3 \mathrm{GHz}$ - Ondřejov \& Zürich radio spectra.) First at 7:30:00 UT a weak X-ray source was observed, then this source splits into two sources
Table 3. Parameters derived from GOES data and RHESSI X-ray spectra at the time of the May 17, 2002 drifting structure observations, $A$ and $V$ are the area and volume of RHESSI X-ray source, $n_{\mathrm{e}}^{\mathrm{GR}}$ and $n_{\mathrm{nt}}^{\mathrm{GR}}$ are the electron plasma and non-thermal densities derived from the GOES emission measure and the RHESSI source volume and spectrum parameters, $n_{\mathrm{e}}^{\mathrm{R}}$ and $n_{\mathrm{nt}}^{\mathrm{R}}$ are the electron plasma and nonthermal densities derived from the RHESSI emission measure and the RHESSI source volume and spectrum parameters, $n_{\mathrm{e}}^{\text {Rad,max }}$ is the electron plasma density derived from the radio spectrum.

\begin{tabular}{lc}
\hline \hline Time (UT) & $7: 38: 22-: 42$ \\
GOES Emission measure $\left(10^{49} \mathrm{~cm}^{-3}\right)$ & 1.0 \\
GOES Temperature $\left(10^{6} \mathrm{~K}\right)$ & 16 \\
\hline RHESSI fit interval $(\mathrm{keV})$ & $3-100$ \\
RHESSI Emission measure $\left(10^{49} \mathrm{~cm}^{-3}\right)$ & 0.56 \\
RHESSI Temperature $\left(10^{6} \mathrm{~K}\right)$ & 20.1 \\
\hline$n_{\mathrm{e}} \times n_{\mathrm{nt}} \times V\left(10^{45} \mathrm{~cm}^{-2} \mathrm{~s}^{-1}\right)$ & 2.64 \\
High energy limit $(\mathrm{keV}))$ & 32000 \\
Power-law index & 5.72 \\
$A\left(10^{17} \mathrm{~cm}^{2}\right)$ & 4.00 \\
\hline$V\left(10^{26} \mathrm{~cm}^{3}\right)$ & 1.9 \\
$n_{\mathrm{e}}^{\mathrm{GR}}(\mathrm{GOES}-\mathrm{RHESSI})\left(10^{11} \mathrm{~cm}^{-3}\right)$ & 2.29 \\
$n_{\mathrm{nt}}^{\mathrm{GR}}(\mathrm{GOES}-\mathrm{RHESSI})\left(10^{7} \mathrm{~cm}^{-3}\right)$ & 6.1 \\
$n_{\mathrm{e}}^{\mathrm{R}}(\mathrm{RHESSI})\left(10^{11} \mathrm{~cm}^{-3}\right)$ & 1.72 \\
$n_{\mathrm{nt}}^{\mathrm{R}}(\mathrm{RHESSI})\left(10^{7} \mathrm{~cm}^{-3}\right)$ & 8.1 \\
$n_{\mathrm{e}}^{\text {Rad,max }}\left(10^{10} \mathrm{~cm}^{-3}\right)$ & 4.00 \\
\hline
\end{tabular}

(see Fig. 6 at 7:32:16 UT and Fig. 7, where the 30-40 keV observation is shown) and then the southern source disappeared and at the beginning of the drifting structure observation one compact source was present (Fig. 6, 7:37:28 UT). The velocity of the source splitting is up to $100 \mathrm{~km} \mathrm{~s}^{-1}$. After 7:36 UT, i.e. at the time of the observation of the slowly drifting structure the X-ray source remained nearly at the same position. On the other hand, above this X-ray source the EIT $195 \AA$ observations show a fast expansion of EIT loops (Fig. 8).

The RHESSI hard X-ray spectrum was analyzed at the beginning of observation of the slowly drifting structure, at 7:38:22-7:38:42 UT. Because of the X-ray source was located above the solar limb, the thin-target model could be used for the spectral fitting. The emission measure and temperature of the X-ray thermal source were derived from GOES as well from RHESSI measurements. The parameters of the fitting are summarized in Table 3. The power-law index of the non-thermal electron distribution was 5.72. Based od the observed X-ray source area $A=4 \times 10^{17} \mathrm{~cm}^{2}$ and estimated source volume $V=$ $1.9 \times 10^{26} \mathrm{~cm}^{3}$ the plasma density $n_{\mathrm{e}}$ and superthermal electron density $n_{\mathrm{nt}}$ in the hard X-ray source were determined as $2.29 \times 10^{11} \mathrm{~cm}^{-3}$ and $6.1 \times 10^{7} \mathrm{~cm}^{-3}$, respectively, using GOES emission measure, and $1.72 \times 10^{11} \mathrm{~cm}^{-3}$ and $8.1 \times 10^{7} \mathrm{~cm}^{-3}$, respectively, using RHESSI emission measure. For comparison the maximum electron plasma density derived from the drifting structure is added $\left(n_{\mathrm{e}}^{\mathrm{Rad}, \max }=4.0 \times 10^{10} \mathrm{~cm}^{-3}\right)$. 

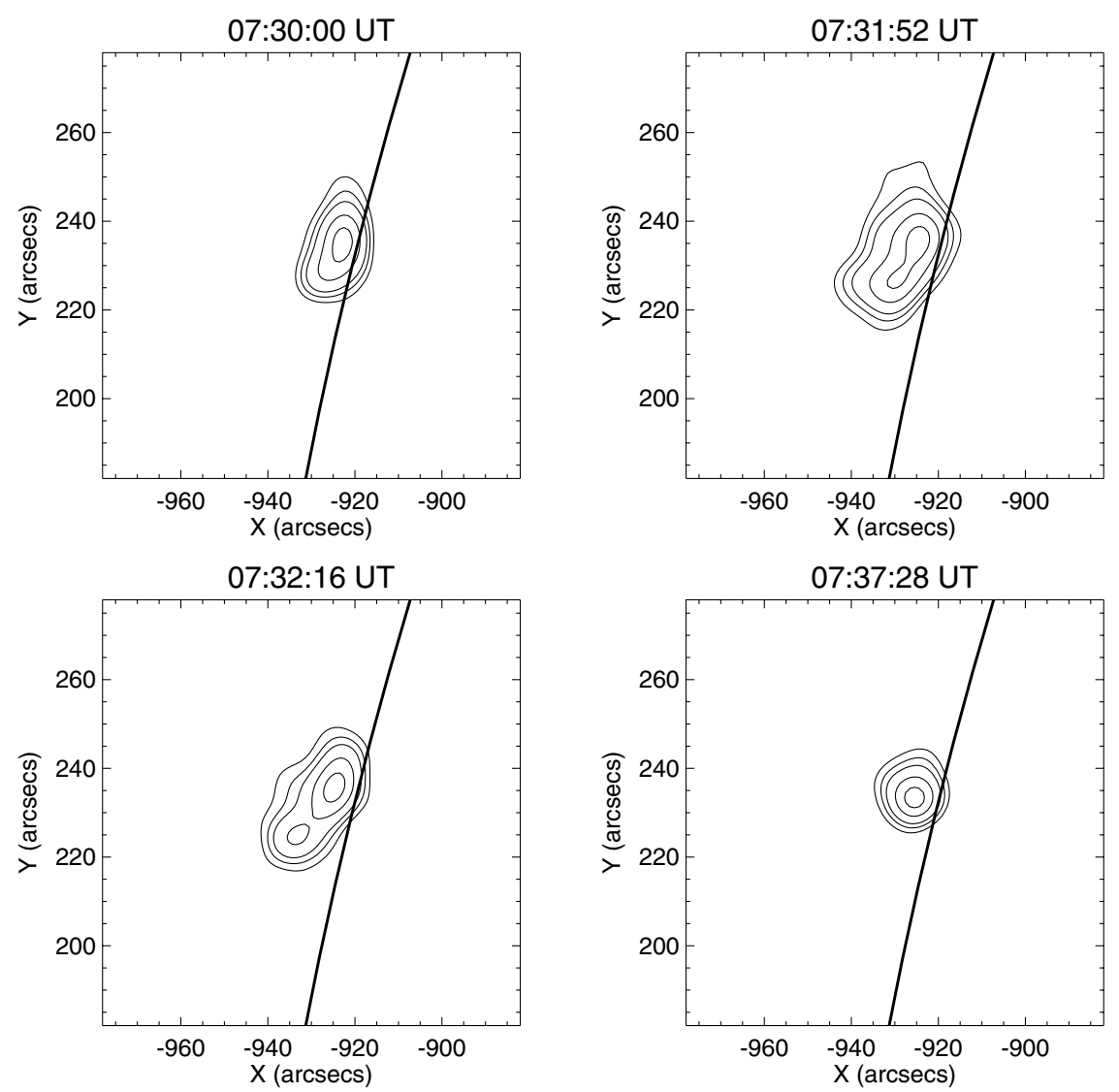

Fig. 6. The RHESSI 6-15 keV image of the X-ray source seen above the north-east solar limb at four times during the May 17, 2002 flare. The contours at 30,40,50,70, $90 \%$ of the maximum flux are shown.

\section{The August 30, 2002 event}

During August 30, 2002, GOES satellite registered at 12:47 UT (start) - 13:29 UT (maximum) - 13:35 UT (end) the soft $\mathrm{X}$-ray event classified as X1.5 flare. The $\mathrm{SN} \mathrm{H} \alpha$ flare has been reported in the AR NOAA 0095 at the position N15E74 at 12:26 UT (start) - 13:28 UT (maximum) - 13:49 UT (end).

On radio waves the high-frequency slowly drifting structure was recorded at 13:27:38-13:27:50 UT in the frequency range $2.0-0.8 \mathrm{GHz}$ (Fig. 10, compare also with GOES, HXRS and RHESSI observations). Its frequency drift was estimated as $-25 \mathrm{MHz} \mathrm{s}^{-1}$ (Table 1). This radio burst was followed by the type II radio burst in the frequency range $100-40 \mathrm{MHz}$ at 13:30-13:40 UT (Potsdam-Tremsdorf observation).

RHESSI imager observed a X-ray source close to the eastnorth solar limb which soft X-ray $(10-20 \mathrm{keV})$ part started to move at the time of the slowly drifting structure observation (Fig. 11, thin-line contours). The source moves systematically in the north-east direction with the velocity in the projection as $10 \mathrm{~km} \mathrm{~s}^{-1}$. For comparison the position of the hard X-ray source $(40-100 \mathrm{keV})$ is shown by the thick-line contours in the image observed at 13:32 UT (Fig. 11).

The RHESSI hard X-ray spectrum was analyzed at the time of the slowly drifting structure, at 13:27:56-13:28:12 UT (Fig. 12). Because the X-ray source was not above the solar limb as in previous cases the X-ray spectrum consists of

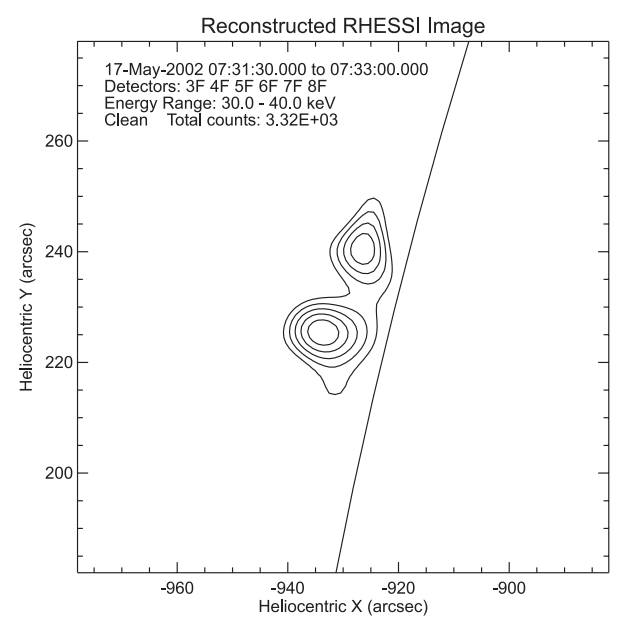

Fig. 7. The $30-40 \mathrm{keV}$ X-ray source observed by RHESSI at 07:31:30-07:33:00 UT, May 17, 2002.

contributions from coronal as well as chromospheric X-ray sources. Therefore, only limited analysis can be done in this case. The emission measure and temperature of the X-ray thermal source were derived as $13.92 \times 10^{49} \mathrm{~cm}^{-3}$ and $21.3 \mathrm{MK}$, respectively. The power-law index of the non-thermal electron distribution was 5.76. The maximum plasma density derived from the drifting structure was $4.94 \times 10^{10} \mathrm{~cm}^{-3}$. 

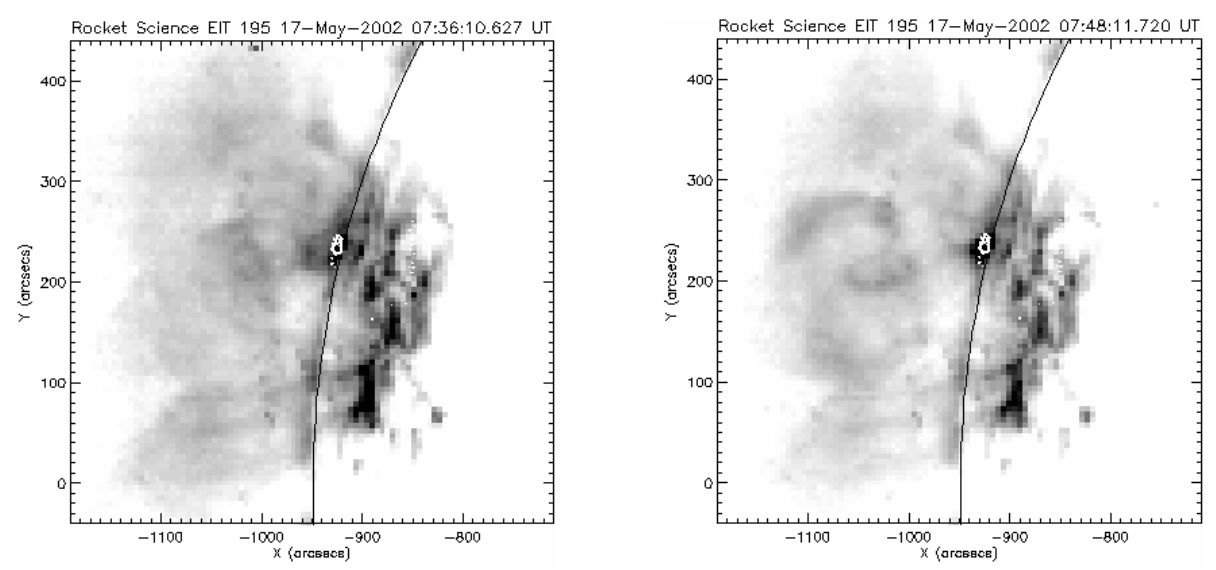

Fig. 8. The EIT $195 \AA$ images of the May 17, 2002 flare at 07:36:10 UT (left) and at 07:48:11 UT (right). The X-ray source in the 6-15 keV energy band at 07:37:14 UT is superimposed by white contours at position $(-925,235)$ arcsecs $(>30 \%$ of maximum).

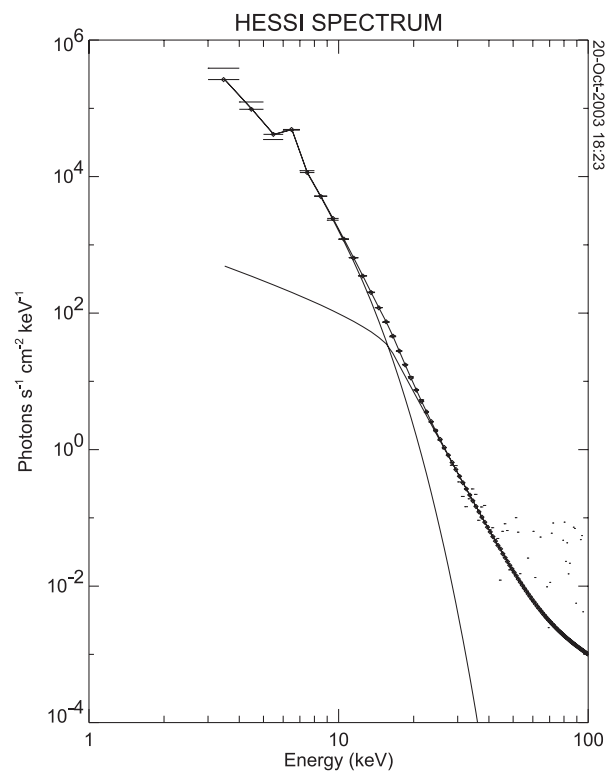

Fig. 9. The RHESSI X-ray spectrum of the hard X-ray source observed during the May 17, 2002 flare at 07:38:22-07:38:41 UT. This spectrum was fitted using the one temperature plus thin-target model of the hard X-ray emission. The resulting spectrum (fitting observed data $=$ $1 \mathrm{keV}$ - long horizontal lines) is a sum of the two components, the thermal and non-thermal ones (two thin lines below the resulting fit).

\section{Correlation of the radio drifting structure and hard X-ray flux observed on August 30, 2002}

Only data from August 30, 2002 event were found to be appropriate for the cross-correlation of the radio flux of the drifting structure with the hard X-ray emission. The drifting structure was integrated along frequencies $(2.0-0.8 \mathrm{GHz})$ and correlated with the 29-44 keV HXRS X-ray flux (Fig. 13). It can be seen that the X-ray emission is partly correlated with an enhanced background of the drifting structure and the X-ray emission is delayed of about $0.5-0.7 \mathrm{~s}$ after the radio. Individual strong radio peaks of the drifting structure are not correlated with the hard X-ray emission.

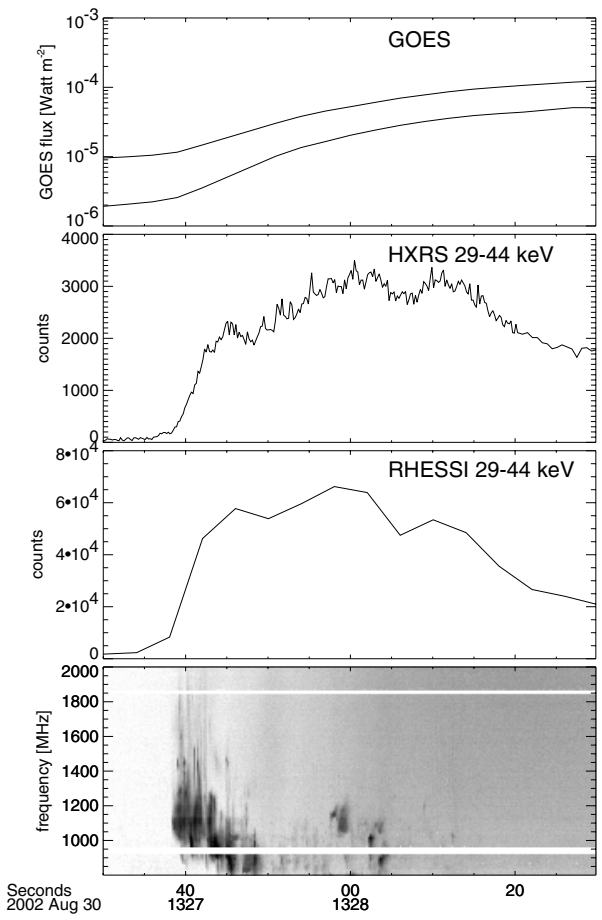

Fig. 10. Time evolution of the August 30, 2002 flare: GOES soft X-ray flux, HXRS 29-44 keV counts, RHESSI 29-44 keV counts, and the $0.8-2.0 \mathrm{GHz}$ radio spectrum showing the slowly drifting structure observed by the Ondřejov radiospectrograph.

\section{Discussion and conclusions}

In three solar flares (April 4, 2002, May 17, 2002, and August 30, 2002) the high-frequency drifting structures were observed simultaneously with the X-ray sources above (April, 4, 2002 and May 17, 2002) and close to the solar limb (August 30, 2002). Comparing basic characteristics of these events it can be seen that the events in April 4, 2002 and August 30, 2002 differs from that in May 17, 2002 in several aspects: a) the drifting structures in April 4 and August 30 are much shorter (32 s and $12 \mathrm{~s}$ ) than that in May 17 event (20 min); b) they have higher frequency drifts; c) in the May 17 event some individual pulses have finite positive frequency drifts 

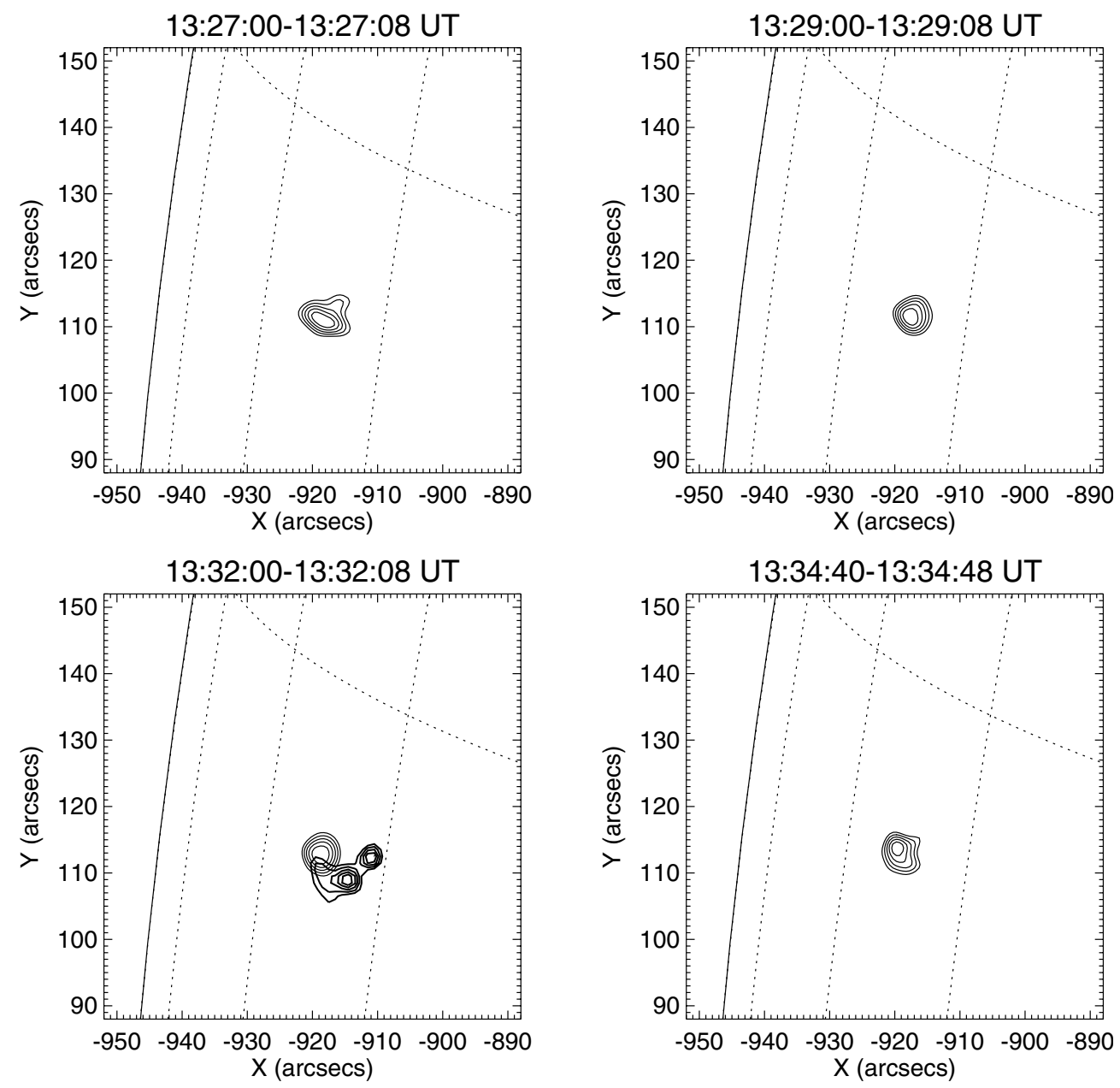

Fig. 11. The RHESSI 10-20 keV image of the X-ray source seen at four times during the August 30, 2002 flare. The position of the 40-100 keV hard X-ray source is shown at 13:32 UT by thick-line contours.

(240-360 $\left.\mathrm{MHz} \mathrm{s}^{-1}\right)$; d) in the April 4 and August 30 event systematic source motions were recorded during and after observations of the drifting structure; but in the May 17 event source splitting into two sources preceded the drifting structure observation; e) the X-ray source in May 17 has much weaker nonthermal component than in other cases. On the other hand, in the August 30, flare the hard X-ray emission up to $300 \mathrm{keV}$ was registered. All these facts show that the events we studied differ in several aspects, but similarities between these events can be recognized too. Moreover, the April 4 and August 30 are similar to those observed in October 5, 1992 (Kliem et al. 2000) and August 18, 1998 (Karlický et al. 2002), and the May 17 event is similar to that observed in November 25, 2000 (Karlický \& Fárník 2003). We think that all the events we studied represent similar physical processes.

As in our previous papers (Kliem et al. 2000; Karlický et al. 2002), we interpret the present high-frequency drifting structures as the plasma radio emission generated by electron beams accelerated during the reconnection and penetrating into the plasmoid magnetic field structure. According to the flare model with the plasmoid ejection (e.g. Ohyama \& Shibata 1998) the reconnection is in the current sheet situated below the ejected plasmoid. But, as can be seen in May 17, 2002 a reality is probably more complex, see the X-ray source splitting at 7:32 UT,
Fig. 6. It is interesting to note that this $\mathrm{X}$-source splitting process was associated with the first hard $\mathrm{X}$-ray and $3 \mathrm{GHz}$ radio bursts, but no radio bursts were recorded below $3 \mathrm{GHz}$. Probably, the acceleration of electrons generating the $\mathrm{dm}$-radio emission (by plasma emission processes) is not strong enough at this initial flare phase or some other conditions for a beam propagation, radio emission and electromagnetic wave propagation were not fulfilled at this time. On the other hand, this source splitting is interesting from the point of view of a formation of several plasmoids in the extended current sheet as proposed by Shibata \& Tanuma (2001). If it was the case, then the current sheet in the May 17, 2002 event was oriented in more or less horizontal direction in comparison with the photosphere. It deviates from the standard model of plasmoid ejection with the vertically oriented current sheet (Shibata \& Tanuma 2001). Another interesting possibility (but akin one) is that we observed during the May 17, 2002 event a rebound of the interacting current-carrying loops in the sense of the paper by Tajima et al. (1987). It can be expected that, after this loops rebound, the current sheet in which the reconnection takes place is formed between these loops (plasmoids). In this sense both possibilities are similar. But in this loop-interacting model the orientation of current sheet can be arbitrary, i.e. in better agreement with observations of the May 17, 2002 event. 


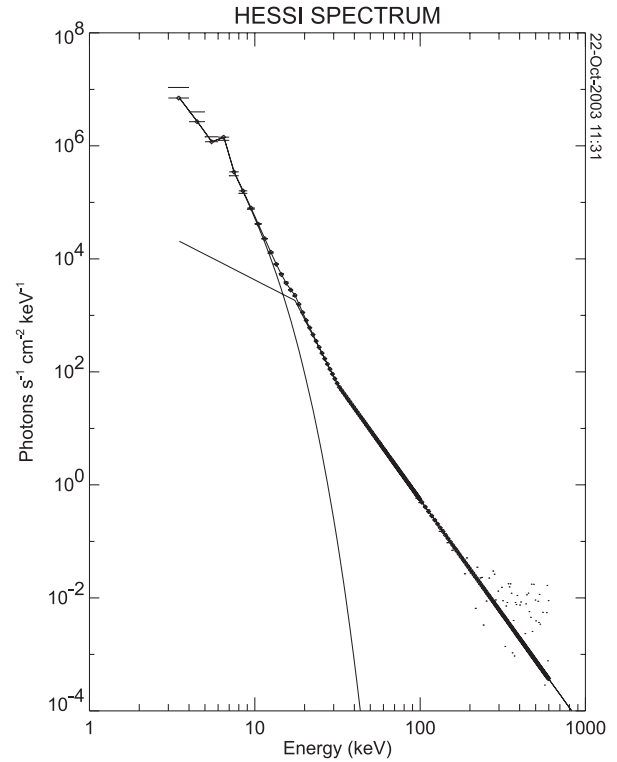

Fig. 12. The RHESSI X-ray spectrum of the hard X-ray source observed during the August 30, 2002 flare at 13:27:56-13:28:12 UT. This spectrum was fitted using the one temperature plus broken powerlaw model of the hard X-ray emission. The resulting spectrum (fitting observed data $=1 \mathrm{keV}$ - long horizontal lines) is a sum of the two components, the thermal and non-thermal ones (two thin lines below the resulting fit).

Furthermore, it is interesting that the coronal X-ray source in the May 17, 2002 event remains nearly at the same position. It agrees with the very slow frequency drift of the drifting structure in this case. But as shown by the EIT observations above this stationary X-ray source the EIT loops expand. This expansion can be confirmed also by the type II burst observation later in the metric range. Probably, similarly as in the April 12, 2001 event (Karlický 2003), both the high-frequency drifting structure and type II radio burst belong to one ejected flare structure. The type II burst is generated in the uppermost part of this structure in super-Alfvenic regime and the high-frequency drifting structure at its lower part in sub-Alfvenic regime.

In the paper by Shibata \& Tanuma (2001) it was shown that an increase of the plasmoid speed means an increase of the reconnection rate. Just this fact can be used for an explanation of differences in the April 4, 2002 and August 30, 2002 events and that one observed on May 17, 2002. The drift rate of the drifting structure observed in May 17, 2002 was much smaller than those observed in the remaining two cases. It means that in this event the reconnection rate was much smaller. It is in agreement with the fact that this event was also much softer in X-ray emission than others. Namely, the reconnection rate is proportional to the electric field $\boldsymbol{E}$ induced in the reconnection process $(\boldsymbol{E} \sim \boldsymbol{v} \times \boldsymbol{B}$, where $\boldsymbol{v}$ is the plasma speed and $\boldsymbol{B}$ is the magnetic field). Thus, a smaller electric field accelerates superthermal electrons with lower energy and softer X-ray sources are generated.

The RHESSI X-ray spectra of all presented cases were fitted by several models. This procedure reveals to be not easy. Nevertheless it was found that the thin-target model was successfully applied only in the case of one coronal X-ray
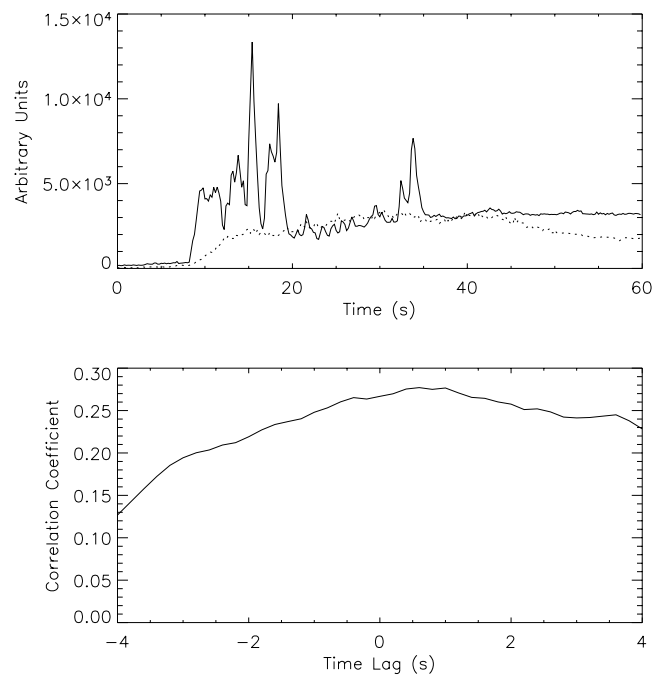

Fig. 13. Top: comparison of the HXRS 29-44 keV X-ray flux (dotted line) and the radio emission flux (solid line) of the slowly drifting structure, integrated over frequencies, observed during the August 30, 2002 flare. The zero time is 13:27:30 UT. Bottom: the corresponding cross-correlation. Positive lag means a delay of hard X-ray emission after the radio.

source (May 17, 2002 case). In more complex cases, with more sources (April 4, 2002 and August 30, 2002) more complex fitting models were necessary.

Comparing the electron densities derived by RHESSI, GOES and radio (April 4, 2002 and May 17, 2002, the cases with the X-ray source above the solar limb) it was found that the density in the radio source of the slowly drifting structure is lower than that estimated in X-ray sources. Based on this fact we can propose that the electron beams, accelerated in the reconnection space near the plasmoid (X-ray source) and generating pulses of the slowly drifting structures, penetrate only into external parts of the plasmoid (due to the O-type magnetic field structure), where the density is lower than in the plasmoid (X-ray source) centre. Namely, numerical simulations show that the plasma density in the reconnection space is lower than that in the plasmoid (Kliem et al. 2000). Later on these electrons diffuse into the plasmoid centre and they increase the X-ray emission as expressed by a time delay $(0.5-0.7 \mathrm{~s})$ found by the cross-correlation method for the August 30, 2002 flare.

It was found that the individual bursts in the drifting structure are not correlated with a relatively smooth X-ray time profile (see Fig. 13). We think that it is due to different emission mechanisms generating dm-radio and X-ray bursts in our case: while the radio emission is generated by strong coherent processes (bump-on-tail instability), X-rays are generated by the bremsstrahlung.

Acknowledgements. This research was supported by Grants IAA3003202, IAA3003003 and S1003006 of the Academy of Sciences, GRANT 205/02/0980 of Grant Agency of the Czech republic and the grant MŠMT ME 550. Authors thank to RHESSI team for data and software. Authors thank an anonymous referee for helpful comments and suggestions. 


\section{References}

Fárník, F., Garcia, H., \& Karlický, M. 2001, Sol. Phys., 201, 357

Hori, K. 1999, in Solar Physics with Radio Observations, Proc. of the Nobeyama Symp., NRO Report No. 479, 267

Hudson, H. S., Kosugi, T., Nitta, N., \& Shimojo, M. 2001, ApJ, 561, L211

Kane, S. R., Sawant, H. S., Cecatto, J. R., et al. 2003, Proc. COSPAR, USA, accepted

Karlický, M. 1998, A\&A, 338, 1084

Karlický, M. 2003, Space Sci. Rev., 107, 81

Karlický, M. 2004, A\&A, in print

Karlický, M., \& Odstrčil, D. 1994, Sol. Phys., 155, 171
Karlický, M., \& Fárník, F. 2003, Adv. Space Res., 32, 2539

Karlický, M., Fárník, F., \& Mészarosová, H. 2002, A\&A, 395, 677

Karlický, M., Kliem, B., Mészárosová, H., \& Jiřička, K. 2002, Proc. of SPM10, Prague, in preparation

Khan, J. I., Vilmer, N., Saint-Hilaire, P., \& Benz, A. O. 2002, A\&A, 388,363

Kliem, B., Karlický, M., \& Benz, A. O. 2000, A\&A, 360, 715

Kundu, M. R., Nindos, A., Vilmer, N., et al. 2001, ApJ, 559, 443

Lin, R. P., Dennis, B. R., Hurford, G. J., et al. 2002, Sol. Phys., 210, 3

Ohyama, M., \& Shibata, K. 1998, ApJ, 499, 934

Shibata, K., \& Tanuma, S. 2001, Earth Planets Space, 53, 473

Tajima, T., Sakai, J., Nakajima, H., et al. 1987, ApJ, 321, 1031 